\title{
ON THE H-FUNCTION
}

\author{
ANATOLY A. KILBAS \\ Belarusian State University \\ Department of Mathematics and Mechanics \\ Minsk 220050, Belarus \\ MEGUMI SAIGO \\ Fukuoka University \\ Department of Applied Mathematics \\ Fukuoka 814-0180, Japan
}

(Received April, 1997; Revised September, 1998)

This paper is devoted to the study of the $H$-function as defined by the Mellin-Barnes integral

$$
H_{p, q}^{m, n}(z)=\frac{1}{2 \pi i} \int_{\mathcal{L}} \mathcal{H}_{p, q}^{m, n}(s) z^{-s} d s
$$

where the function $J_{p, q}^{m, n}(s)$ is a certain ratio of products of the Gammafunctions with the argument $s$ and the contour $\mathcal{L}$ specially chosen. The conditions for the existence of $H_{p, q}^{m, n}(z)$ are discussed and explicit power and power-logarithmic series expansions of $H_{p, q}^{m, n}(z)$ near zero and infinity are given. The obtained results define more precisely the known results.

Key words: $H$-Function.

AMS subject classifications: $33 \mathrm{C} 40$.

\section{Introduction}

This paper deals with the $H$-function $H_{p, q}^{m, n}(z)$ introduced by Pincherle in 1888 (see [3, Section 1.19]). Interest in this function appeared in 1961, when Fox [4] investigated such a function as a symmetrical Fourier kernel. Therefore, the $H$ function is often called Fox's $H$-function. For integers $m, n, p$ and $q$ such that $0 \leq m \leq q$ and $0 \leq n \leq p$, while $a_{i}, b_{j} \in \mathbb{C}$ and $\alpha_{i}, \beta_{j} \in \mathbb{R}_{+}=(0, \infty)$, with $1 \leq i \leq p$ and $1 \leq j \leq q$, the function is defined by the Mellin-Barnes integral:

where

$$
H_{p, q}^{m, n}(z)=\frac{1}{2 \pi i} \int_{\mathcal{L}} \mathfrak{H}_{p, q}^{m, n}(s) z^{-s} d s,
$$




$$
\mathcal{H}_{p, q}^{m, n}(s)=\frac{\prod_{j=1}^{m} \Gamma\left(b_{j}+\beta_{j} s\right) \prod_{i=1}^{n} \Gamma\left(1-a_{i}-\alpha_{i} s\right)}{\prod_{i=n+1}^{p} \Gamma\left(a_{i}+\alpha_{i} s\right) \prod_{j=m+1}^{q} \Gamma\left(1-b_{j}-\beta_{j} s\right)} .
$$

The contour $\mathcal{L}$ is specially chosen and an empty product, if it occurs, is taken to be one. The theory of this function may be found in $[1,2,9$ Chapter 2, 10, 11 Section 8.3] and [12, Chapter 1]. We only indicate that most of the elementary and special functions are particular cases of the $H$-function $H_{p, q}^{m, n}(z)$. In particular, if $\alpha$ 's and $\beta$ 's are all equal to 1 , the $H$-function (1.1) reduces to Meijer's $G$-function $G_{p, q}^{m, n}(z)$.

The conditions of the existence of the $H$-function can be made by inspecting the convergence of the integral (1.1), which depends on the selection of the contour $\mathcal{L}$ and on the relations between parameters $a_{i}$ and $\alpha_{i}(i=1, \ldots, p)$ and $b_{j}$ and $\beta_{j}(j=$ $1, \ldots, q)$. Especially, the relations might depend on the numbers $\Delta, \delta$ and $\mu$ defined by

$$
\begin{gathered}
\Delta=\sum_{j=1}^{q} \beta_{j}-\sum_{i=1}^{p} \alpha_{i}, \\
\delta=\prod_{i=1}^{p} \alpha_{i}^{-\alpha_{i}} \prod_{j=1}^{q} \beta_{j}^{\beta_{j}}, \\
\mu=\sum_{j=1}^{q} b_{j}-\sum_{i=1}^{p} a_{i}+\frac{p-q}{2} .
\end{gathered}
$$

Such a selection of $\mathcal{L}$ and various relations between parameters are indicated in the handbook [11, Section 8.3.1]; but some of the results there need correction. In this paper we would like to give such corrections in the following cases.

(a) $\quad \Delta \geq 0$ and the contour $\mathcal{L}=\mathcal{L}_{-\infty}$ in (1.1) runs from $-\infty+i \varphi_{1}$ to $-\infty+$ $i \varphi_{2}$, with $\varphi_{1}<\varphi_{2}$, such that the poles of the Gamma-functions of the form $\Gamma\left(b_{j}+\beta_{j} s\right)(j=1, \ldots, m)$ lie on the left of $\mathcal{L}{ }_{-\infty}$ and those of the functions of the form $\Gamma\left(1-a_{i}-\alpha_{i} s\right)(i=1, \ldots, n)$ lie on the right of $\mathcal{L}_{-\infty}$.

(b) $\quad \Delta \leq 0$ and the contour $\mathcal{L}=\mathcal{L}+\infty$ in (1.1) runs from $+\infty+i \varphi_{1}$ to $+\infty+$ $i \varphi_{2}$, with $\varphi_{1}<\varphi_{2}$, such that the poles of the Gamma-functions of the form $\Gamma\left(b_{j}+\beta_{j} s\right)(j=1, \ldots, m)$ lie on the left of $\mathcal{L}_{+\infty}$ and those of the functions of the form $\Gamma\left(1-a_{i}-\alpha_{i} s\right)(i=1, \ldots, n)$ lie on the right of $\mathcal{L}_{+\infty}$.

Our results are based on the asymptotic behavior of the function ${ }^{+}{ }_{p, q}^{\infty} m(s)$, given in (1.2) at infinity. Using the behavior and following [1], we give the series representation of $H_{p, q}^{m, n}(z)$ via residues of the integrand $\mathfrak{H}_{p, q}^{m, n}(s) z^{-s}$. In this way, we simplify the proof of Theorem 1 in [1] by applying the former results to find the explicit series expansions of $H_{p, q}^{m, n}(z)$. Such power expansions, as corollaries of the results from [1], were indicated in [12, Chapter 2.2] (see also [11, Section 8.3.1]), provided that the poles of the Gamma-functions of the forms $\Gamma\left(b_{j}+\beta_{j} s\right)$ $(j=1, \ldots, m)$ and $\Gamma\left(1-a_{i}-\alpha_{i} s\right)(i=1, \ldots, n)$ do not coincide, that is,

$$
\beta_{j}\left(a_{i}-1-k\right) \neq \alpha_{i}\left(b_{j}+l\right) \quad\left(i=1, \ldots, n ; j=1, \ldots, m ; k, l \in \mathbb{N}_{0}=\{0,1,2, \ldots\}\right)
$$

in the following cases. 
(c) $\Delta>0$ with $z \neq 0$ or $\Delta=0$ with $0<|z|<\delta$, and the poles of the Gamma-functions of the form $\Gamma\left(b_{j}+\beta_{j} s\right)(j=1, \ldots, m)$ are simple:

$$
\beta_{j}\left(b_{i}+k\right) \neq \beta_{i}\left(b_{j}+l\right) \quad\left(i \neq j ; i, j=1, \ldots, m ; k, l \in \mathbb{N}_{0}\right)
$$

(d) $\Delta<0$ with $z \neq 0$ or $\Delta=0$ with $|z|>\delta$, and the poles of the Gammafunctions of the form $\Gamma\left(1-a_{i}-\alpha_{i} s\right)(i=1, \ldots, n)$ are simple:

$$
\alpha_{j}\left(1-a_{i}+k\right) \neq \alpha_{i}\left(1-a_{j}+l\right) \quad\left(i \neq j ; i, j=1, \ldots, n ; k, l \in \mathbb{N}_{0}\right) .
$$

When the poles of the Gamma-functions in (c) and (d) coincide, explicit series expansions of $H_{p, q}^{m, n}(z)$ should be more complicated power-logarithmic expansions. Such expansions in particular cases of the Meijer's $G$-functions, $G_{0, p}^{p, 0}$ and $G_{p, p}^{p, 0}$, and of the $H$-functions, $H_{0, p}^{p, 0}$ and $H_{p, p}^{p, 0}$, were given in [7] and [8], respectively.

We obtain explicit expansions of the $H$-function of general form $H_{p, q}^{m, n}(z)$ under the conditions in (1.6). We show that, if the poles of the Gamma-functions of the forms $\Gamma\left(b_{j}+\beta_{j} s\right)(j=1, \ldots, m)$ and $\Gamma\left(1-a_{i}-\alpha_{i} s\right)(i=1, \ldots, n)$ coincide in the cases (c) and (d), respectively, then the $H$-function (1.1) has power-logarithmic series expansions. In particular, we give asymptotic expansions of $H_{p, q}^{m, n}(z)$ near zero. We note that the obtained results will be different in the cases when either $\Delta \geq 0$ or $\Delta \leq 0$.

The paper is organized as follows. Section 2 is devoted to the conditions of the existence of the $H$-function (1.1) which are based on the asymptotic behavior of $\mathcal{K}_{p, q}^{m, n}(s)$ at infinity. Here we also give the representations of (1.1) via the residues of the integrand. The latter result is applied in Sections 3 and 4 to obtain the explicit power and power-logarithmic series expansions of $H_{p, q}^{m, n}(z)$ and its asymptotic estimates near zero.

\section{Existence and Representations of $H_{p, q}^{m, n}(z)$}

First, we give the asymptotic estimate of the Gamma-function $\Gamma(z), z=x+i y,[3$, Chapter 1] at infinity on lines parallel to the coordinate axes.

Lemma 1: Let $z=x+i y$ with $x, y \in \mathbb{R}=(-\infty, \infty)$. Then the following asymptotic estimates at infinity are valid

$$
\begin{gathered}
|\Gamma(x+i y)| \sim \sqrt{2 \pi}|x|^{x-1 / 2} e^{-x-\pi(1-\operatorname{sign} x) y / 2} \\
(|x| \rightarrow \infty ; y \neq 0 \text { if } x<0)
\end{gathered}
$$

and

$$
|\Gamma(x+i y)| \sim \sqrt{2 \pi}|y|^{x-1 / 2} e^{-x-\pi|y| / 2} \quad(|y| \rightarrow \infty) .
$$

Proof: Applying the Stirling formula [3, 1.18(2)],

we have

$$
\Gamma(z) \sim \sqrt{2 \pi} e^{(z-1 / 2) \log (z)} e^{-z} \quad(|z| \rightarrow \infty ;|\arg (z)|<\pi),
$$

$$
\begin{gathered}
|\Gamma(x+i y)| \sim \sqrt{2 \pi} \mid e^{(x+i y-1 / 2)[\log |x+i y|+i \arg (x+i y)]} e^{-(x+i y) \mid} \\
\sim \sqrt{2 \pi}|x+i y|^{x-1 / 2} e^{-x-y \arg (x+i y)} \quad(|x+i y| \rightarrow \infty ; y \neq 0 \text { if } x \leq 0) .
\end{gathered}
$$


Let $y \in \mathbb{R}$ be fixed and $|x| \rightarrow \infty$. Then $|x+i y| \sim|x|$, and $\arg (x+i y) \rightarrow 0$ as $x \rightarrow+\infty$ and $\arg (x+i y) \rightarrow \pi$ as $x \rightarrow-\infty$. Therefore, (2.4) implies

and

$$
|\Gamma(x+i y)| \sim \sqrt{2 \pi}|x|^{x-1 / 2} e^{-x} \quad(x \rightarrow+\infty)
$$

$$
|\Gamma(x+i y)| \sim \sqrt{2 \pi}|x|^{x-1 / 2} e^{-x-\pi y} \quad(x \rightarrow-\infty ; y \neq 0),
$$

which yield (2.1).

Turning to the case where $x \in \mathbb{R}$ is fixed and $|y| \rightarrow \infty$, we find $|x+i y| \sim|y|$ and $\arg (x+i y) \rightarrow \pi / 2$ as $y \rightarrow \infty$, and $\arg (x+i y) \rightarrow-\pi / 2$ as $y \rightarrow-\infty$. Thus $(2.4)$ implies (2.2).

Remark 1: In view of (2.2), the relation [3, (1.18.6)] needs correction with addition of the multiplier $e^{x}$ on the left-hand side and it must be replaced by

$$
\lim _{|y| \rightarrow \infty}|\Gamma(x+i y)| e^{x+\pi|y| / 2}|y|^{1 / 2-x}=\sqrt{2 \pi} .
$$

The next assertion gives the asymptotic behavior of $\mathfrak{J}_{p, q}^{m, n}(s)$, defined in (1.2), at infinity on lines parallel to the real axis.

Lemma 2: Let $\Delta, \delta$ and $\mu$ be defined as in (1.3) to (1.5) and let $t, \sigma \in \mathbb{R}$. Then the following estimates hold:

with

$$
\left|\mathcal{J}_{p, q}^{m, n}(t+i \sigma)\right| \sim A\left(\frac{e}{t}\right)^{-\Delta t} \delta^{t} t^{\operatorname{Re}(\mu)} \quad(t \rightarrow+\infty)
$$

$$
\begin{aligned}
& A=(2 \pi)^{m+n-(p+q) / 2} e^{q-m-n}
\end{aligned}
$$

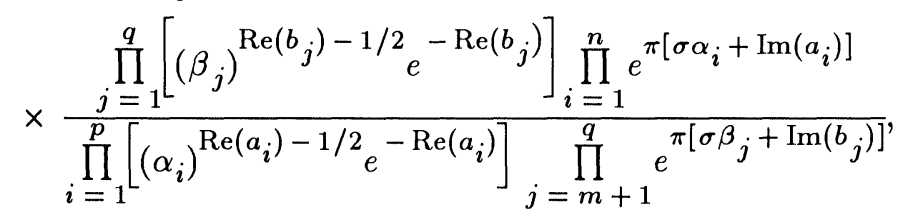

and

$$
\left|\mathcal{H}_{p, q}^{m, n}(t+i \sigma)\right| \sim B\left(\frac{e}{|t|}\right)^{\Delta|t|} \delta-|t||t|^{\operatorname{Re}(\mu)} \quad(t \rightarrow-\infty)
$$

with

$$
\begin{aligned}
& B=(2 \pi)^{m+n-(p+q) / 2} e^{q-m-n}
\end{aligned}
$$

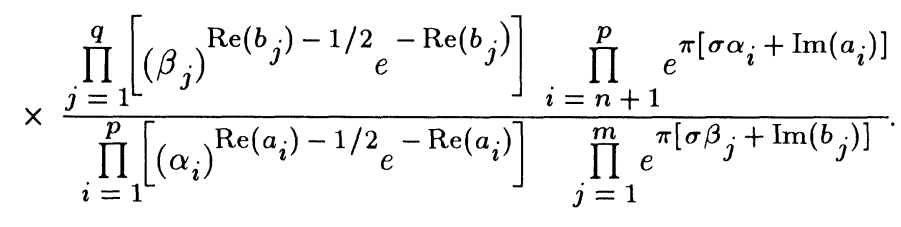

Proof: By virtue of (2.1), we have, for a variable $s=t+i \sigma$ and a complex constant $k=c+i d$,

and

$$
|\Gamma(s+k)| \sim \sqrt{2 \pi} t+c-1 / 2 e^{-(t+c)} \quad(t \rightarrow+\infty)
$$

$$
|\Gamma(s+k)| \sim \sqrt{2 \pi}|t|^{t+c-1 / 2} e^{-(t+c)} e^{-\pi(\sigma+d)} \quad(t \rightarrow-\infty)
$$


Substituting these estimates into (1.2) and using (1.3) to (1.5), we obtain (2.8) and (2.10).

Remark 2: The asymptotic estimate of the function ${ }^{3} \mathcal{F}_{p, q}^{m, n}(s)$ at infinity on lines parallel to the imaginary axis $\mathfrak{H}_{p, q}^{m, n}(\sigma+i t)$ as $|t| \rightarrow \infty$ was given in our paper with Shlapakov [5].

By appealing to Lemma 2, we give conditions of the existence of the $H$-function (1.1) with the contour $\mathcal{L}$ being chosen as indicated in (a) and (b) in Section 1.

Theorem 1: Let $\Delta, \delta$ and $\mu$ be given by (1.3) to (1.5). Then the function $H_{p, q}^{m, n}(z)$ defined by (1.1) and (1.2) exists in the following cases:

$$
\begin{gathered}
\mathcal{L}=\mathcal{L}_{-\infty}, \Delta>0, \quad z \neq 0 ; \\
\mathcal{L}=\mathcal{L}_{-\infty}, \quad \Delta=0, \quad 0<|z|<\delta ; \\
\mathcal{L}=\mathcal{L}_{-\infty}, \quad \Delta=0, \quad|z|=\delta, \quad \operatorname{Re}(\mu)<-1 ; \\
\mathcal{L}=\mathcal{L}_{+\infty}, \quad \Delta<0, \quad z \neq 0 ; \\
\mathcal{L}=\mathcal{L}_{+\infty}, \quad \Delta=0, \quad|z|>\delta ; \\
\mathcal{L}=\mathcal{L}_{+\infty}, \quad \Delta=0, \quad|z|=\delta, \operatorname{Re}(\mu)<-1 .
\end{gathered}
$$

Proof: Let us first consider the case (a) of Section 1 for which $\Delta \geq 0$ and $\mathcal{L}=\mathcal{L}_{-\infty}$. We have to investigate the convergence of the integral (1.1) on the lines

$$
l_{1}=\left\{t \in \mathbb{R}: t+i \varphi_{1}\right\} \text { and } l_{2}=\left\{t \in \mathbb{R}: t+i \varphi_{2}\right\} \text { for } \varphi_{1}<\varphi_{2}
$$

as $t \rightarrow-\infty$. According to $(2.10)$, we have the following asymptotic estimate for the integrand of (1.1):

$$
\begin{aligned}
\left|\mathcal{H}_{p, q}^{m, n}(s) z^{-s}\right| & \sim B_{i} e^{\varphi_{i} \arg z}\left(\frac{e}{|t|}\right)^{\Delta|t|}\left(\frac{|z|}{\delta}\right)^{|t|}|t|^{\operatorname{Re}(\mu)} \\
& \left(t \rightarrow-\infty ; \quad t \in l_{i}(i=1,2)\right),
\end{aligned}
$$

where $B_{1}$ and $B_{2}$ are given by (2.11) with $\sigma$ being replaced by $\varphi_{1}$ and $\varphi_{2}$, respectively. It follows from (2.21) that the integral (1.1) is convergent if and only if one of the conditions (2.14) to (2.16) is satisfied.

In the case (b), $\Delta \leq 0$ and the contour $\mathcal{L}$ is taken to be $\mathcal{L}+\infty$. So we have to investigate the convergence of the integral (1.1) on the lines $l_{1}$ and $l_{2}$ in $(2.20)$ as $t \rightarrow$ $+\infty$. By virtue of (2.8) and (2.9), we have the asymptotic estimate:

$$
\begin{gathered}
\left|\mathcal{J}_{p, q}^{m, n}(s) z^{-s}\right| \sim A_{i} e^{\varphi_{i} \arg z}\left(\frac{e}{t}\right)^{-\Delta t}\left(\frac{\delta}{|z|}\right)^{t} t^{\operatorname{Re}(\mu)} \\
\left(t \rightarrow+\infty ; \quad t \in l_{i}(i=1,2)\right)
\end{gathered}
$$

where $A_{1}$ and $A_{2}$ are given by (2.9) with $\sigma$ being replaced by $\varphi_{1}$ and $\varphi_{2}$, respectively. Thus (2.22) implies that the integral (1.1) converges if and only if one of the conditions (2.17) to (2.19) holds.

Corollary 1: The estimate (2.21) holds for $t \rightarrow-\infty$ uniformly on sets which have 
a positive distance to each point,

$$
b_{j l}=-\frac{b_{j}+l}{\beta_{j}} \quad\left(j=1, \ldots, m ; l \in \mathbb{N}_{0}\right)
$$

and which do not contain points to the right of $\mathcal{L}_{-\infty}$.

The estimate (2.22) holds for $t \rightarrow+\infty$ uniformly on sets which have a positive distance to each point,

$$
a_{i k}=\frac{1-a_{i}+k}{\alpha_{i}} \quad\left(i=1, \ldots, n ; k \in \mathbb{N}_{0}\right)
$$

and which do not contain points to the left of $\mathcal{L}_{+\infty}$.

Remark 3: In [11, Section 8.3.1], for the existence of the $H$-function (1.1) the conditions,

$$
\sum_{i=1}^{n} \alpha_{i}-\sum_{i=n+1}^{p} \alpha_{i}+\sum_{j=1}^{m} \beta_{j}-\sum_{j=m+1}^{q} \beta_{j} \geq 0 \text { and } \operatorname{Re}(\mu)<0
$$

are given in the cases when $\mathcal{L}=\mathcal{L}_{-\infty}, \Delta=0$ and $|z|=\delta$ and when $\mathcal{L}=\mathscr{L}_{+\infty}$, $\Delta=0$ and $|z|=\delta$. But according to (2.16) and (2.19), (2.25) can be replaced by the condition,

$$
\operatorname{Re}(\mu)<-1
$$

The next statement follows from Theorem 1, Corollary 1 and the theory of residues.

Theorem 2: (A) If the conditions in (1.6) and (2.14) or (2.15) are satisfied, then the H-function (1.1) is an analytic function of $z$ in the corresponding domain indicated in (2.14) or (2.15), and

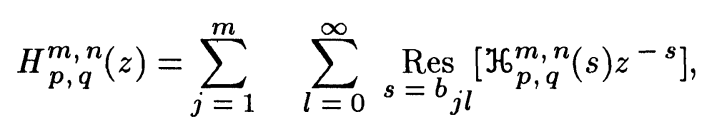

where each $b_{j l}$ is given in (2.23).

(B) If the conditions in (1.6) and (2.17) or (2.18) are satisfied, then the $H$ function (1.1) is an analytic function of $z$ in the corresponding domain indicated in (2.17) or (2.18), and

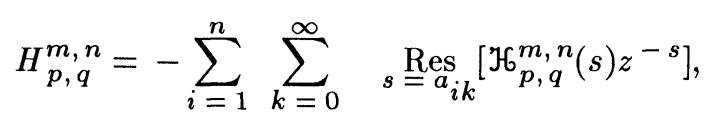

where each $a_{i k}$ is given in (2.24).

Remark 4: Theorem 2(A) was proved in [1, p. 278, Theorem 1] for the $H$ function represented by the integrand obtained from (1.1) and (1.2) after replacing $s$ by $-s$. It sproof is rather complicated and is based on a lemma, where the asymptotic estimate at infinity of the function $h_{0}(s)$ defined by

$$
h_{0}(s)=\frac{\prod_{i=1}^{p} \Gamma\left(1-a_{i}+\alpha_{i} s\right)}{\prod_{j=1}^{q} \Gamma\left(1-b_{j}+\beta_{j} s\right)}
$$

is given. But our proof of Theorem 2 along the ideas of [1] is simpler and is based on the asymptotic estimate of $\mathcal{H}_{p, q}^{m, n}(s)$ itself at infinity given in Lemma 2. 


\section{Explicit Power Series Expansions}

In this section we apply Theorem 2 to obtain explicit power series expansions of the $H$-function (1.1) under the conditions of (1.6) in the cases of (1.7) and (1.8), individually.

First, we consider the former case. By Theorem 2(A), we have to evaluate the residue of $\mathcal{H}(s) z^{-s}$ at each point $s=b_{j l}$ given in (2.23); in what follows we simplify $\mathcal{J}_{p, q}^{m, n}(s)$ by $\mathcal{H}_{\mathcal{C}}(s)$. To evaluate these residues we use the property of the Gammafunction $[6,(3.30)]$, that is, in a neighborhood of the pole $z=-k\left(k \in \mathbb{N}_{0}\right)$, the Gamma-function $\Gamma(z)$ can be expanded in powers of $z+k=\epsilon$ so that

$$
\Gamma(z)=\frac{(-1)^{k}}{k ! \epsilon}\left[1+\epsilon \psi(1+k)+O\left(\epsilon^{2}\right)\right], \text { where } \psi(z)=\frac{\Gamma^{\prime}(z)}{\Gamma(z)} .
$$

Since each pole $b_{j l}$ is simple, i.e., the conditions in (1.7) hold,

$$
\operatorname{Res}_{s=b}\left[\mathfrak{H} G(s) z^{-s}\right]=h_{j l}^{*} z^{-b} j l \quad\left(j=1, \ldots, m ; l \in \mathbb{N}_{0}\right),
$$

where

$$
\begin{gathered}
h_{j l}^{*}=\lim _{s \rightarrow b_{j l}}\left[\left(s-b_{j l}\right) \mathfrak{H}(s)\right] \\
=\frac{(-1)^{l} \prod_{i=1, i \neq j}^{m} \Gamma\left(b_{i}-\left[b_{j}+l\right] \frac{\beta_{i}}{\beta_{j}}\right) \prod_{i=1}^{n} \Gamma\left(1-a_{i}+\left[b_{j}+l\right] \frac{\alpha_{i}}{\beta_{j}}\right)}{l ! \beta_{j} \prod_{n+1}^{p} \Gamma\left(a_{i}-\left[b_{j}+l\right] \frac{\alpha_{i}}{\beta_{j}}\right) \prod_{i=m+1}^{q} \Gamma\left(1-b_{i}+\left[b_{j}+l\right] \frac{\beta_{i}}{\beta_{j}}\right)} .
\end{gathered}
$$

Thus we obtain the following theorem.

Theorem 3: Let the conditions (1.6) and (1.7) be satisfied and let either $\Delta>0$ and $z \neq 0$ or $\Delta=0$ and $0<|z|<\delta$. Then the $H$-function (1.1) has the power series expansion

$$
H_{p, q}^{m, n}(z)=\sum_{j=1}^{m} \sum_{l=0}^{\infty} h_{j l}^{*} z^{\left(b_{j}+l\right) / \beta_{j}}
$$

where each constant $h_{j l}^{*}$ is given by (3.3).

Corollary 2: If the conditions (1.6) and (1.7) are satisfied and $\Delta \geq 0$, then (3.4) gives the asymptotic expansion of $H_{p, q}^{m, n}(z)$ near zero, and the main terms of this asymptotic formula have the form:

$$
H_{p, q}^{m, n}(z)=\sum_{j=1}^{m}\left[h_{j}^{*} z^{b_{j} / \beta_{j}}+O\left(z^{\left(b_{j}+1\right) / \beta_{j}}\right)\right] \quad(z \rightarrow 0),
$$

where

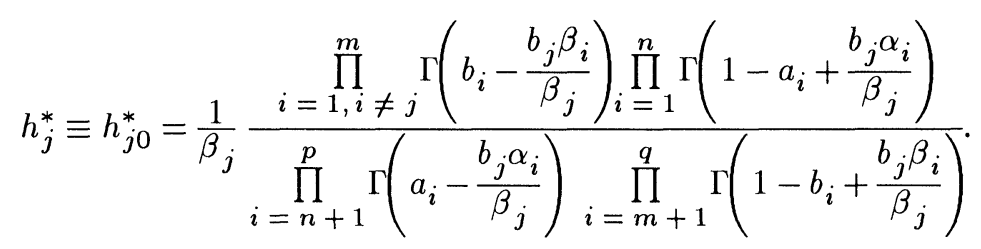


Corollary 3: Let the conditions (1.6) and (1.7) be satisfied, and let $\Delta \geq 0$ and $j_{0}$ $\left(1 \leq j_{0} \leq m\right)$ be an integer such that

$$
\frac{\operatorname{Re}\left(b_{j_{0}}\right)}{\beta_{j_{0}}}=\min _{1 \leq j \leq m}\left[\frac{\operatorname{Re}\left(b_{j}\right)}{\beta_{j}}\right]
$$

Then there holds the asymptotic estimate:

$$
H_{p, q}^{m, n}(z)=h_{j_{0}}^{*} z^{b j_{0} / \beta} j_{0}+o\left(z^{b_{0} / \beta} j_{0}\right) \quad(z \rightarrow 0)
$$

where $h_{j_{0}}^{*}$ is given by (3.6) with $j=j_{0}$. In particular,

$$
H_{p, q}^{m, n}(z)=O\left(z^{\rho^{*}}\right) \quad(z \rightarrow 0) \text { with } \rho^{*}=\min _{1 \leq j \leq m}\left[\frac{\operatorname{Re}\left(b_{j}\right)}{\beta_{j}}\right] .
$$

Now we consider the case (1.8) when the poles of the Gamma-functions of the form $\Gamma\left(1-a_{i}-\alpha_{i} s\right)(i=1, \ldots, n)$ are simple. By (3.1), evaluating the residues of $\mathfrak{J}(s) z^{-s}$ considering each point $a_{i k}$ given in (2.24), we have similarly to the previous argument that

$$
s \stackrel{\operatorname{Res}}{=} a_{i k}\left[\mathfrak{H}(s) z^{-s}\right]=-h_{i k} z^{-a_{i k}} \quad\left(i=1, \ldots, n ; k \in \mathbb{N}_{0}\right),
$$

where $a_{i k}$ is given by $(2.24)$ and

$$
\begin{gathered}
h_{i k}=\lim _{s \rightarrow a_{i k}}\left[-\left(s-a_{i k}\right) \mathcal{H}(s)\right] \\
=\frac{(-1)^{k}}{k ! \alpha_{i}} \frac{\prod_{j=1}^{m} \Gamma\left(b_{j}+\left[1-a_{i}+k\right] \frac{\beta j}{\alpha_{i}}\right)}{\prod_{j=1}^{p} \Gamma\left(a_{j}+\left[1-a_{i}+k\right] \frac{\alpha_{j}}{\alpha_{i}}\right)} \Gamma \prod_{j=m+1}^{n} \Gamma\left(1-a_{j}-\left[1-a_{i}+k\right] \frac{\alpha_{j}}{\alpha_{i}}\right) \\
\end{gathered}
$$

Thus from Theorem 2(B) we have the following theorem.

Theorem 4: Let the conditions (1.6) and (1.8) be satisfied and let either $\Delta<0$ and $z \neq 0$ or $\Delta=0$ and $|z|>\delta$. Then the $H$-function (1.1) has the power series expansion:

$$
H_{p, q}^{m, n}(z)=\sum_{i=1}^{n} \sum_{k=0}^{\infty} h_{i k} z^{\left(a_{i}-k-1\right) / \alpha_{i}}
$$

where each constant $h_{i k}$ is given by (3.11).

Corollary 4: If the conditions (1.6) and (1.8) are satisfied and $\Delta \leq 0$, then (3.12) gives the asymptotic expansion of $H_{p, q}^{m, n}(z)$ near infinity, and the main terms of this asymptotic formula have the form:

$$
H_{p, q}^{m, n}(z)=\sum_{i=1}^{n}\left[h_{i} z^{\left(a_{i}-1\right) / \alpha_{i}}+O\left(z^{\left.\left(a_{i}-2\right) / \alpha_{i}\right)}\right] \quad(|z| \rightarrow \infty)\right.
$$

where

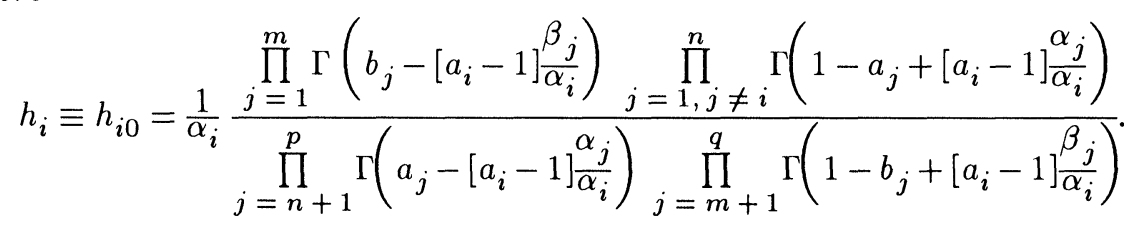


Corollary 5: Let the conditions (1.6) and (1.8) be satisfied, and let $\Delta \leq 0$ and $i_{0}$ $\left(1 \leq i_{0} \leq n\right)$ be an integer such that

$$
\frac{\operatorname{Re}\left(a_{i_{0}}\right)-1}{\alpha_{i_{0}}}=\max _{1 \leq i \leq n}\left[\frac{\operatorname{Re}\left(a_{i}\right)-1}{\alpha_{i}}\right]
$$

Then there holds the asymptotic estimate:

$$
H_{p, q}^{m, n}(z)=h_{i_{0}} z^{\left(a_{i_{0}}-1\right) / \alpha_{i}}+o\left(z^{\left(a_{i_{0}}-1\right) / \alpha_{i_{0}}}\right) \quad(|z| \rightarrow \infty)
$$

where $h_{i_{0}}$ is given by (3.14) with $i=i_{0}$. In particular,

$$
H_{p, q}^{m, n}(z)=O\left(z^{\varrho}\right) \quad(|z| \rightarrow \infty) \text { with } \varrho \underset{1}{=} \max _{\leq i \leq n}\left[\frac{\operatorname{Re}\left(a_{i}\right)-1}{\alpha_{i}}\right] \text {. }
$$

\section{Explicit Power-Logarithmic Series Expansions}

Now, let us discuss cases when the conditions in (1.6) hold, but either (1.7) or (1.8) is violated.

(e) $\quad \mathcal{L}=\mathcal{L}_{-\infty}, \Delta \geq 0$ and some poles of the Gamma-functions of the form $\Gamma\left(b_{j}+\beta_{j} s\right)(j=1, \ldots, m)$ coincide.

(f) $\quad \mathcal{L}=\mathcal{L}+\infty, \Delta \leq 0$ and some poles of the Gamma-functions of the form $\Gamma\left(1-a_{i}-\alpha_{i} s\right)(i=1, \ldots, n)$ coincide.

First, we consider the case (e). Let $b \equiv b_{j l}$ be one of points (2.23) for which some poles of the Gamma-functions of the form $\Gamma\left(b_{j}+\beta_{j} s\right)(j=1, \ldots, m)$ coincide and let $N^{*} \equiv N_{j l}^{*}$ be the order of a coinciding pole. Then there must exist $j_{1}, \ldots, j_{N^{*}} \in$ $\{1, \ldots, m\}$ and $l_{j_{1}}, \ldots, l_{j^{*}} \in \mathbb{N}_{0}$ such that

$$
b \equiv-\frac{{ }^{b} j_{1}+l}{\beta_{j_{1}}}=\ldots=-\frac{{ }^{b}{ }_{j_{1}^{*}}+l_{j_{N^{*}}}}{\beta_{j_{N^{*}}}} .
$$

Then $\mathcal{H}(s) z^{-s}$ has the pole of order $N^{*}$ at $b$ and hence

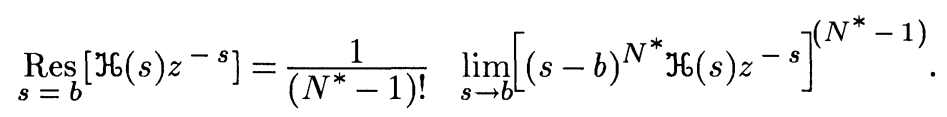

We denote

$$
\begin{aligned}
& \mathfrak{H}_{1}^{*}(s)=(s-b)^{N^{*}} \prod_{j=j_{1}}^{N^{*}} \Gamma\left(b_{j}+\beta_{j} s\right) \text { and } \mathfrak{H}_{2}^{*}(s)=\frac{\mathfrak{H}(s)}{\prod_{j=j_{1}}^{N^{*}} \Gamma\left(b_{j}+\beta_{j} s\right)} . \\
& \text { Leibniz rule, we have }
\end{aligned}
$$

Using the Leibniz rule, we have

$$
\begin{aligned}
& {\left[(s-b)^{N^{*}} \mathfrak{H G}(s) z^{-s}\right]^{\left(N^{*}-1\right)}=\sum_{n=0}^{N^{*}-1}\left(\begin{array}{c}
N^{*}-1 \\
n
\end{array}\right)\left[\mathcal{G G}_{1}^{*}(s)\right]^{\left(N^{*}-1-n\right)}\left[\mathcal{H G}_{2}^{*}(s) z^{-s}\right]^{(n)}} \\
& =\sum_{n=0}^{N^{*}-1}\left(\begin{array}{c}
N^{*}-1 \\
n
\end{array}\right)\left[\mathcal{H} \mathcal{G}_{1}^{*}(s)\right]^{\left(N^{*}-1-n\right)} \sum_{i=0}^{n}\left(\begin{array}{c}
n \\
i
\end{array}\right)(-1)^{i}\left[\mathcal{H}_{2}^{*}(s)\right]^{(n-i)} z^{-s}[\log (z)]^{i}
\end{aligned}
$$




$$
=z^{-s} \sum_{i=0}^{N^{*}-1}\left\{\sum_{n=i}^{N^{*}-1}(-1)^{i}\left(\begin{array}{c}
N^{*}-1 \\
n
\end{array}\right)\left(\begin{array}{l}
n \\
i
\end{array}\right)\left[\mathcal{H}_{1}^{*}(s)\right]^{\left(N^{*}-1-n\right)}\left[\mathfrak{H}_{2}^{*}(s)\right]^{(n-i)}\right\}[\log (z)]^{i}
$$

Substituting this into (4.2), we obtain

where

$$
\operatorname{Res}_{s=b_{j l}}\left[\mathfrak{H}(s) z^{-s}\right]=z^{\left(b_{j}+l\right) / \beta} j \sum_{i=0}^{N_{j l}^{*}-1} H_{j l i}^{*}[\log (z)]^{i}
$$

$$
\begin{gathered}
H_{j l i}^{*} \equiv H_{j l i}^{*}\left(N_{j l}^{*} ; b_{j l}\right) \\
=\frac{1}{\left(N_{j l}^{*}-1\right) !} \sum_{n=i}^{N_{j l}^{*}-1}(-1)^{i}\left(\begin{array}{c}
N_{j l}^{*}-1 \\
n
\end{array}\right)\left(\begin{array}{c}
n \\
i
\end{array}\right)\left[\mathcal{H}_{1}^{*}\left(b_{j l}\right)\right]^{\left(N_{j l}^{*}-1-n\right)}\left[\mathcal{H}_{2}^{*}\left(b_{j l}\right)\right]^{(n-i)}
\end{gathered}
$$

In particular, if $l=0$ and $i=N_{j 0}^{*}-1$, then by setting $N_{j 0}^{*} \equiv N_{j}^{*}$ and using (3.1), (4.1) and (4.3), we have

$$
\begin{aligned}
& H_{j}^{*}=H_{j, 0, N_{j}^{*}-1}^{*}\left(N_{j}^{*} ; b_{j 0}\right)=\frac{(-1)^{N_{j}^{*}-1}}{\left(N_{j}^{*}-1\right) !} \mathcal{H}_{1}^{*}\left(b_{j 0}\right) \mathcal{H}_{2}^{*}\left(b_{j 0}\right)
\end{aligned}
$$

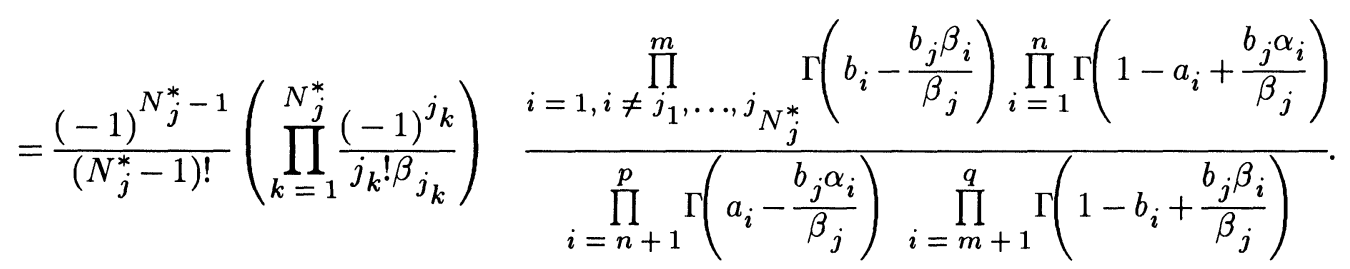

Thus, in view of Theorem 2(A), we have the following.

Theorem 5: Let the conditions in (1.6) be satisfied and let either $\Delta>0$ and $z \neq 0$ or $\Delta=0$ and $0<|z|<\delta$. Then the $H$-function (1.1) has the power-logarithmic series expansion:

$$
H_{p, q}^{m, n}(z)=\sum_{j, l}^{\prime} h_{j l}^{*} z^{\left(b_{j}+l\right) / \beta_{j}}+\sum_{j, l}{ }_{i=0}^{N} \sum_{j l}^{*} H_{j l i}^{*} z^{\left(b_{j}+l\right) / \beta} j[\log (z)]^{i}
$$

Here $\sum^{\prime}$ and $\sum^{\prime \prime}$ are summations taken over $j$ and $l\left(j=1, \ldots, m ; l \in \mathbb{N}_{0}\right)$ such that the Gamma-functions of the form $\Gamma\left(b_{j}+\beta_{j} s\right)$ have simple poles and poles of order $N_{j l}^{*}$ at the points of the form $b_{j l}$ respectively, while each constant $h_{j l}^{*}$ is given by (3.3) and each constant $H_{j l i}^{*}$ is given by (4.5).

Corollary 6: If the conditions in (1.6) are satisfies and $\Delta \geq 0$, then (4.7) gives the asymptotic expansion of $H_{p, q}^{m, n}(z)$ near zero, and the main terms of this asymptotic formula have the form:

$$
\begin{gathered}
H_{p, q}^{m, n}(z)=\sum_{j=1}^{m}{ }^{\prime}\left[h_{j}^{*} z^{b_{j} / \beta_{j}}+O\left(z^{\left(b_{j}+1\right) / \beta_{j}}\right)\right] \\
+\sum_{j=1}^{m}{ }^{\prime \prime}\left[H_{j}^{*} z^{b^{b} / \beta_{j}}[\log (z)]^{N_{j}^{*}-1}+O\left(z^{\left(b_{j}+1\right) / \beta_{j}}[\log (z)]^{\left.N_{j}^{*}-1\right)}\right) \quad(z \rightarrow 0) .\right.
\end{gathered}
$$

Here $\Sigma^{\prime}$ and $\Sigma^{\prime \prime}$ are summations taken over $j(j=1, \ldots, m)$ such that the Gamma- 
functions of the form $\Gamma\left(b_{j}+\beta_{j} s\right)$ have simple poles and poles of order $N_{j}^{*} \equiv N_{j 0}^{*}$ at the points of the form $b_{j 0}$ respectively, while each $h_{j}^{*}$ is given by (3.6) and each $H_{j}^{*}$ is given by (4.6).

Corollary 7: Let the conditions in (1.6) be satisfied, and let $\Delta \geq 0$ and $b_{j l}$ be the pole of the Gamma-function of the form $\Gamma\left(b_{j}+\beta_{j} s\right)(j=1, \ldots, m)$. Let $j_{01}$ and $j_{02}$ $\left(1 \leq j_{01}, j_{02} \leq m\right)$ be integers such that

$$
\rho_{1}^{*} \equiv \frac{\operatorname{Re}\left(b_{j_{01}}\right)}{\beta_{j_{01}}}=\min _{1 \leq j \leq m}\left[\frac{\operatorname{Re}\left(b_{j}\right)}{\beta_{j}}\right]
$$

when the pole of the form $b_{j l} \quad\left(j=1, \ldots, m ; l \in \mathbb{N}_{0}\right)$ is simple, and

$$
\rho_{2}^{*} \equiv \frac{\operatorname{Re}\left(b_{j_{02}}\right)}{\beta_{j_{02}}}=\min _{1 \leq j \leq m}\left[\frac{\operatorname{Re}\left(b_{j}\right)}{\beta_{j}}\right]
$$

when the poles of the form $b_{j l}\left(j=1, \ldots, m ; l \in \mathbb{N}_{0}\right)$ coincide.

(a) If $\rho_{1}^{*}<\rho_{2}^{*}$, then the asympotitc expansion of the $H$-function has the form:

$$
H_{p, q}^{m, n}(z)=h_{j_{01}}^{*} z^{b j_{01} / \beta} j_{01}+o\left(z^{{ }^{b} j_{01} / \beta} j_{01}\right) \quad(z \rightarrow 0)
$$

where $h_{j_{01}}^{*}$ is given by (3.6) with $j=j_{01}$. In particular, the relation (3.9) holds.

(b) If $\rho_{1}^{*} \geq \rho_{2}^{*}$ and $b_{j_{02}, 0}$ is the pole of order $N_{j_{02}}^{*}$, then the asymptotic expan sion of the $H$-function has the form:

$$
\begin{gathered}
H_{p, q}^{m, n}(z)=H_{j_{02}}^{*} z^{b_{j_{02}} / \beta_{j}}{ }_{02}[\log (z)]^{N_{j_{02}}^{*}-1}+o\left(z^{b_{j_{02} / \beta_{j}}}{ }^{\left.-1 \log (z)]^{N_{j_{02}}^{*}-1}\right)}\right. \\
(z \rightarrow 0),
\end{gathered}
$$

where $H_{j_{02}}^{*}$ is given by (4.6) with $j=j_{02}$. In particular, if $N^{*}$ is the largest order of general poles of the Gamma-functions of the form $\Gamma\left(b_{j}+\beta_{j} s\right)(j=1, \ldots, m)$, then

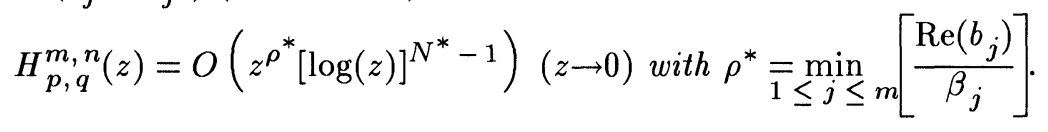

Now we consider the case (f). Let $a=a_{i k}$ be one of points (2.24) for which some poles of the Gamma-functions of the form $\Gamma\left(1-a_{i}-\alpha_{i} s\right)$ coincide and let $N=N_{i k}$ be the order of this common pole. Then there exist $i_{1}, \ldots, i_{N} \in\{1, \ldots, n\}$ and $k_{i_{1}}, \ldots, k_{i_{N}} \in \mathbb{N}_{0}$ such that

$$
a \equiv \frac{1-a_{i_{1}}+k_{i_{1}}}{\alpha_{i_{1}}}=\ldots=\frac{1-a_{i_{N}}+k_{i_{N}}}{\alpha_{i_{N}}} .
$$

Thus the integrand $\mathfrak{H}(s) z^{-s}$ of the integrand (1.1) has the pole of order $N$ at $a$. Similarly to (4.3), we denote

$$
\mathfrak{H}_{1}(s)=(s-a)^{N} \prod_{i=i_{1}}^{i} \Gamma\left(1-a_{i}-\alpha_{i} s\right) \text { and } \mathfrak{H}_{2}(s)=\frac{\mathfrak{H}(s)}{\prod_{i=i_{1}}^{i_{1}} \Gamma\left(1-a_{i}-\alpha_{i} s\right)}
$$


and, then, we find similarly to (4.4) and (4.5) that

where

$$
s \stackrel{\operatorname{Res}}{=}\left[\mathcal{H} a_{i k}(s) z^{-s}\right]=z^{\left(a_{i}-1-k\right) / \alpha_{i}} \sum_{j=0}^{N_{i k}-1} H_{i k j}[\log (z)]^{j}
$$

$$
\begin{gathered}
H_{i k j} \equiv H_{i k j}\left(N_{i k} ; a_{i k}\right) \\
=\frac{1}{\left(N_{i k}-1\right) !} \sum_{n=j}^{N_{i k}^{-1}}(-1)^{j}\left(\begin{array}{c}
N_{i k}-1 \\
n
\end{array}\right)\left(\begin{array}{c}
n \\
j
\end{array}\right)\left[\mathfrak{H}_{1}\left(a_{i k}\right)\right]^{\left(N_{i k}-1-n\right)}\left[\mathcal{H}_{2}\left(a_{i k}\right)\right]^{(n-j)} .
\end{gathered}
$$

In particular, if we set $k=0, j=N_{i 0}-1$ and $N_{i 0} \equiv N_{i}$, then, using (4.15) and (3.1), we have

$$
\begin{aligned}
& H_{i} \equiv H_{i, 0, N_{i}-1}\left(N_{i} ; a_{i 0}\right)=\frac{(-1)^{N_{i}-1}}{\left(N_{i}-1\right) !} \mathfrak{H}_{1}\left(a_{i 0}\right) \mathfrak{H G}_{2}\left(a_{i 0}\right)=\frac{(-1)^{N_{i}-1}}{\left(N_{i}-1\right) !}
\end{aligned}
$$

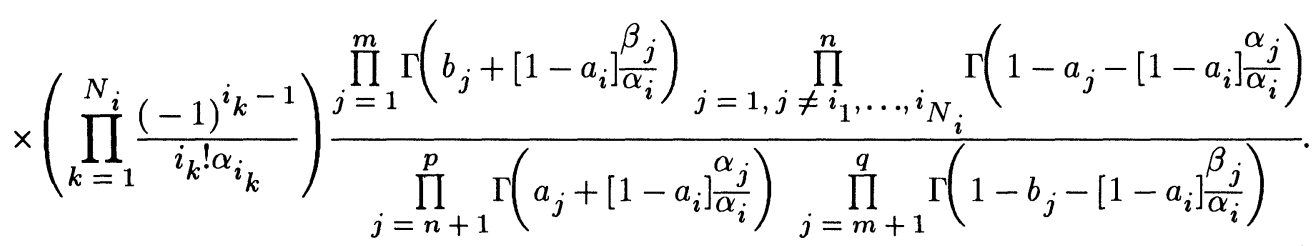

Therefore, Theorem 2(B) implies the following result similar to Theorem 5 .

Theorem 6: Let the conditions in (1.6) be satisfied and let either $\Delta<0$ and $z \neq 0$ or $\Delta=0$ and $|z|>\delta$. Then the H-function (1.1) has the power-logarithmic series expansion:

$$
H_{p, q}^{m, n}(z)=\sum_{i, k}^{\prime} h_{i k} z^{\left(a_{i}-1-k\right) / \alpha_{i}}+\sum_{i, k}^{\prime \prime} \sum_{j=0}^{N_{i k}-1} H_{i k j} z^{\left(a_{i}-1-k\right) / \alpha_{i}}[\log (z)]^{j}
$$

Here $\sum^{\prime}$ and $\sum^{\prime \prime}$ are summations taken over $i$ and $k\left(i=1, \ldots, n ; k \in \mathbb{N}_{0}\right)$ such that the Gamma-functions of the form $\Gamma\left(1-a_{i}-\alpha_{i} s\right)$ have simple poles and poles of order $N_{i k}$ at the points of the form $a_{i k}$ respectively, while each constant $h_{i k}$ is given by (3.11) and each constant $H_{i k j}$ is given by (4.17).

Corollary 8: If the conditions in (1.6) are satisfied and $\Delta \leq 0$, then (4.19) gives the asymptotic expansion of $H_{p, q}^{m, n}(z)$ near infinity, and the main terms of this asymptotic formula have the form:

$$
\begin{gathered}
H_{p, q}^{m, n}(z)=\sum_{i=1}^{n}{ }^{\prime}\left[h_{i} z^{\left(a_{i}-1\right) / \alpha_{i}}+O\left(z^{\left(a_{i}-2\right) / \alpha_{i}}\right)\right] \\
+\sum_{i=1}^{n}{ }^{\prime \prime}\left[H_{i} z^{\left(a_{i}-1\right) / \alpha_{i}}[\log (z)]^{N_{i}-1}+O\left(z^{\left(a_{i}-2\right) / \alpha_{i}}[\log (z)]^{N_{i}-1}\right)\right](|z| \rightarrow \infty) .
\end{gathered}
$$

Here $\sum^{\prime}$ and $\sum^{\prime \prime}$ are summations taken over $i(i=1, \ldots, n)$ such that the Gammafunctions of the form $\Gamma\left(1-a_{i}-\alpha_{i} s\right)$ have simple poles and poles of order $N_{i} \equiv N_{i 0}$ at the points of the form $a_{i 0}$ in (2.24) respectively, while each $h_{i}$ is given by $(3.14)$ 
and each $H_{i}$ is given by (4.18).

Corollary 9: Let the conditions in (1.6) be satisfied, and let $\Delta \leq 0$ and $a_{i k}$ be the pole of the Gamma-function of the form $\Gamma\left(1-a_{i}-\alpha_{i} s\right)(i=1, \ldots, n)$. Let $i_{01}$ and $i_{02}$ $\left(1 \leq i_{01}, i_{02} \leq n\right)$ be integers such that

$$
\rho_{1} \equiv \frac{\operatorname{Re}\left(a_{i_{01}}\right)-1}{\alpha_{i_{01}}}=\max _{1 \leq i \leq n}\left[\frac{\operatorname{Re}\left(a_{i}\right)-1}{\alpha_{i}}\right]
$$

when the pole of the form $a_{i k}\left(i=1, \ldots, n ; k \in \mathbb{N}_{0}\right)$ is simple, and

$$
\rho_{2} \equiv \frac{\operatorname{Re}\left(a_{i_{02}}\right)-1}{\alpha_{i_{02}}}=\max _{1 \leq i \leq n}\left[\frac{\operatorname{Re}\left(a_{i}\right)-1}{\alpha_{i}}\right]
$$

when the poles of the form $a_{i k}\left(i=1, \ldots, n ; k \in \mathbb{N}_{0}\right)$ coincide.

(a) If $\rho_{1}>\rho_{2}$, then the asymptotic expansion of the $H$-function has the form:

$H_{p, q}^{m, n}(z)=h_{i_{01}} z^{\left(a_{i_{01}}-1\right) / \alpha_{i_{01}}}+o\left(z^{\left(a_{i_{01}}-1\right) / \alpha_{i}}\right) \quad(|z| \rightarrow \infty)$,

where $h_{i_{01}}$ is given by (3.14) with $i=i_{01}$. In particular, the relation (3.17) holds.

(b) If $\rho_{1} \leq \rho_{2}$ and $a_{i_{02}, 0}$ is the pole of order $N_{i_{02}}$, then asymptotic expansion of the H-function has the form:

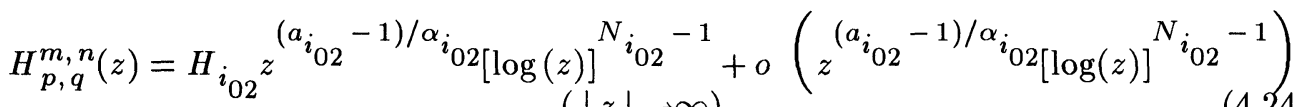

$$
\begin{aligned}
& (|z| \rightarrow \infty)
\end{aligned}
$$

where $H_{i_{02}}$ is given by (4.18) with $i=i_{02}$. In particular, if $N$ is the smallest order of general poles of the Gamma-functions of the form $\Gamma\left(1-a_{i}-\alpha_{i} s\right)(i=1, \ldots, n)$, then

$$
H_{p, q}^{m, n}(z)=O\left(z^{\varrho}[\log (z)]^{N-1}\right)(|z| \rightarrow \infty) \text { with } \varrho=\max _{1 \leq i \leq n}\left[\frac{\operatorname{Re}\left(a_{i}\right)-1}{\alpha_{i}}\right]
$$

In conclusion, we give the following consequence of Corollaries $3,5,7$ and 9, which unifies the power and power-logarithmic asymptotic behavior of $H_{p, q}^{m, n}(z)$ near zero and infinity.

Theorem 7: Let the conditions in (1.6) be satisfied.

(a) If $\Delta \geq 0$ and the poles of the Gamma-functions of the form $\Gamma\left(b_{j}+\beta_{j} s\right)$ $(j=1, \ldots, m)$ are simple, then the $H$-function (1.1) has the asymptotic estimate (3.9) at zero. If some of the poles of the Gamma-functions of the form $\Gamma\left(b_{j}+\beta_{j} s\right)(j=1, \ldots, m)$ coincide, then $H_{p, q}^{m, n}(z)$ has the asymptotic estimate either (3.9) or (4.13) at zero.

(b) If $\Delta \leq 0$ and the poles of the Gamma-functions of the form $\Gamma\left(1-a_{i}-\alpha_{i} s\right)$ $(i=1, \ldots, n)$ are simple, then the $H$-function (1.1) has the asymptotic estimate (3.17) at infinity. If some of the poles of the Gamma-functions of the form $\Gamma\left(1-a_{i}-\alpha_{i} s\right)(i=1, \ldots, n)$ coincide, then $H_{p, q}^{m, n}(z)$ has the asymptotic estimate (3.17) or (4.25) at infinity.

Remark 5: The power-logarithmic expansions and more complicated results than those in (4.7) were indicated in [9, Section 3.7] (see also [8, Section 5.8]); and the 
particular cases, $H_{0, p}^{p, 0}(z)$ and $H_{p, p}^{p, 0}(z)$, are shown in [7].

\section{Acknowledgement}

The authors would like to express their gratitude to the referee for valuable comments.

\section{References}

[1] Braaksma, B.L.J., Asymptotic expansions and analytic continuations for a class of Barnes-integrals, Compositio Math. 15 (1964), 239-341.

[2] Dixon, A.L. and Ferrar, W.L., A class of discontinuous integrals, Quart. J. Math., Oxford Ser. 7 (1936), 81-96.

[3] Erdélyi, A., Magnus, W., Oberhettinger, F. and Tricomi, F.G., Higher Transcendental Functions, Vol. I, McGraw-Hill, New York-Toronto-London 1953.

[4] Fox, C., The $G$ and $H$ functions as symmetrical Fourier kernels, Trans. Amer. Math. Soc. 98 (1961), 395-429.

[5] Kilbas, A.A., Saigo, M. and Shlapakov, S.A., Integral transforms with Fox's $H$ function in spaces of summable functions, Integral Transform. Spec. Funct. 1 (1993), 87-103.

[6] Marichev, O.I., Handbook of Integral Transforms of Higher Transcendental Function. Theory and Algorithmic Tables, Ellis Horwood, Chichester; John Wiley \& Sons, New York 1983.

[7] Mathai, A.M., An expansion of Meijer's $G$-function in the logarithmic case with applications, Math. Nachr. 48 (1971), 129-139.

[8] Mathai, A.M., A few remarks on the exact distributions of certain multivariate statistics II, Multivariate Statistical Inferences (Proc. Res. Sem., Dalhousie Univ., Halifax, N.S. 1972), 169-181, North-Holland, Amsterdam and New York 1973.

[9] Mathai, A.M. and Saxena, R.K., The H-Function with Applications in Statistics and Other Disciplines, Halsted Press (John Wiley \& Sons), New York, London, Sydney 1978.

[10] Mellin, Hj., Abriss einer einheitlichen Theorie der Gamma- und der hypergeometrischen Funktionen, Math. Ann. 68 (1910), 305-337.

[11] Prudnikov, A.P., Brychkov, Yu.A. and Marichev, O.I., Integrals and Series, Vol. 3, More Special Functions, Gordon and Breach, New York-PhiladelphiaLondon-Paris-Montreux-Tokyo-Melbourne 1990.

[12] Srivastava, H.M., Gupta, K.C. and Goyal, S.P., The H-Functions of One and Two Variables with Applications, South Asian Publishers, New Delhi 1982. 


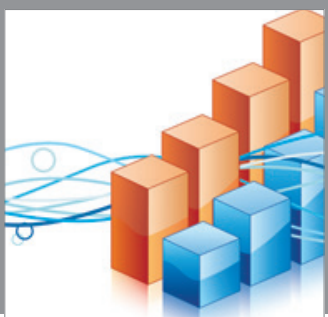

Advances in

Operations Research

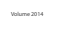

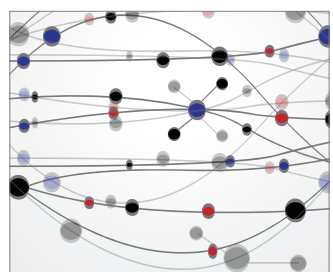

\section{The Scientific} World Journal
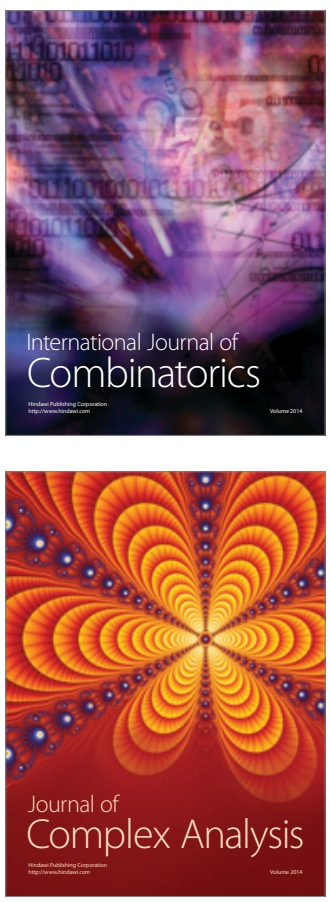

International Journal of

Mathematics and

Mathematical

Sciences
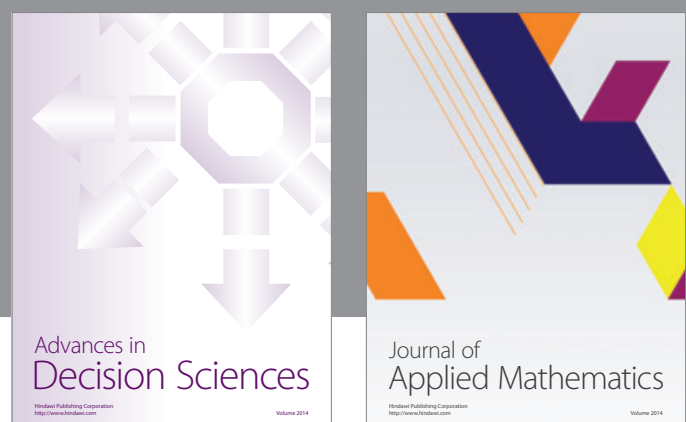

Journal of

Applied Mathematics
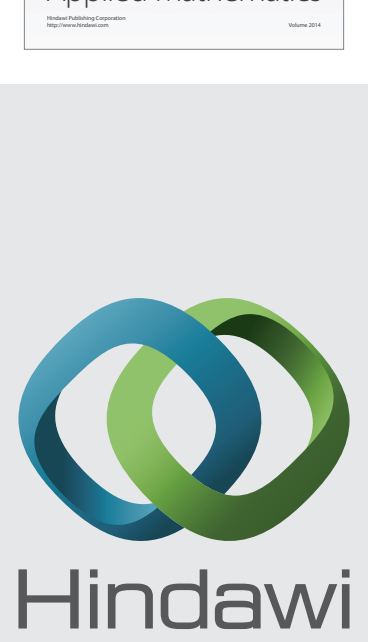

Submit your manuscripts at http://www.hindawi.com
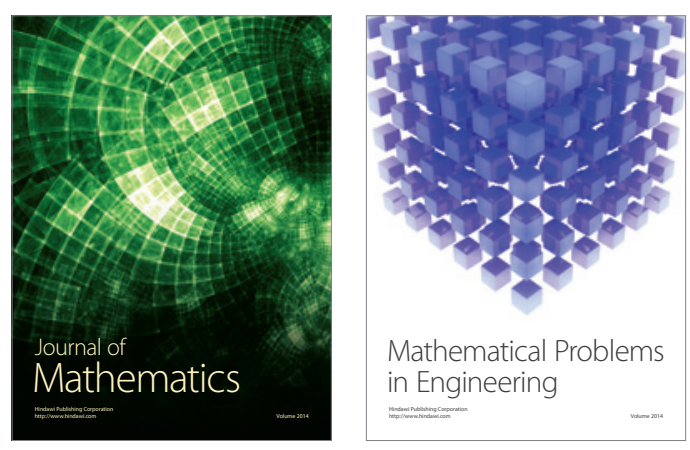

Mathematical Problems in Engineering
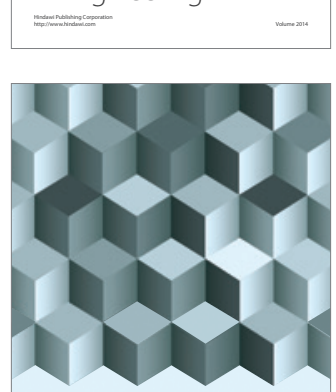

Journal of

Function Spaces
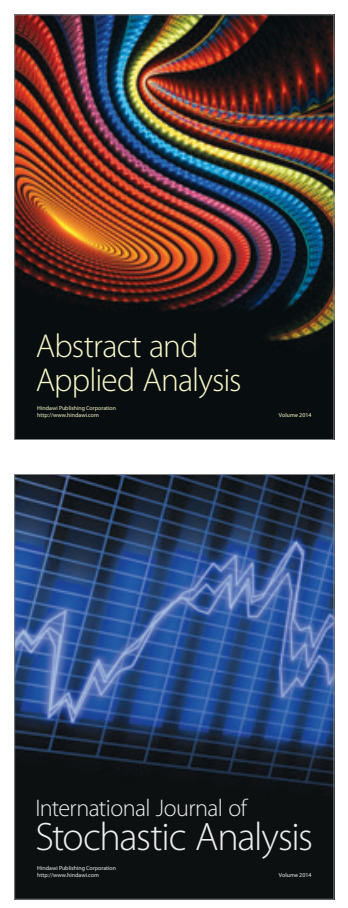

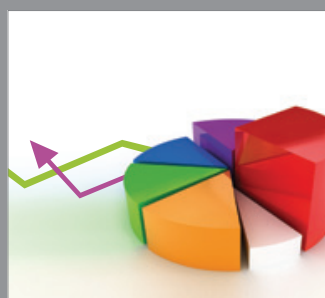

ournal of

Probability and Statistics

Promensencen
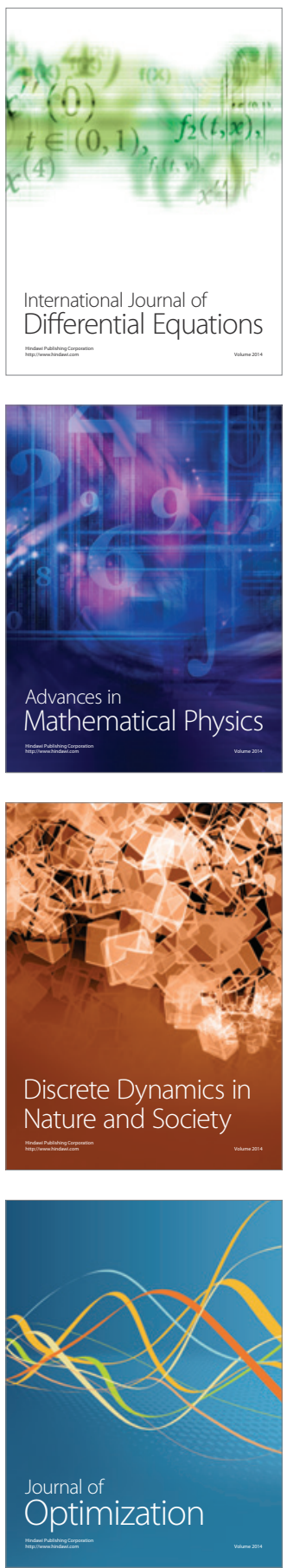